\title{
Evaluación del efecto de la asociación de coberturas vege- tales vivas sobre el cultivo de uvilla (Physalis peruviana L.) en Huaca, provincia del Carchi, Ecuador
}

\section{Assessment of the effect of crop live coverage association on golden berry crop (Physalis peruviana L.) in Huaca, Carchi province, Ecuador}

\author{
Carlos David Herrera Ramírez ${ }^{1}$ Jaime Ramiro Hidrobo Luna ${ }^{2}$, Emilio Rodrigo Basantes Morales ${ }^{3}$ \\ ${ }^{1}$ Universidad Politécnica Estatal del Carchi. Calle Antisana y Av. Universitaria. Tulcán, Ecuador. \\ Calle Antisana s/n y Av. Universitaria. Tulcán, Ecuador \\ ${ }^{2}$ Universidad Central del Ecuador. Facultad de Ciencias Agrícolas. \\ Jerónimo Leiton y Av. La Gasca s/n. Ciudadela Universitaria. Quito. 170521. Ecuador \\ ${ }^{3}$ Universidad de las Fuerzas Armadas. Campus Universitario.Departamento de Ciencias de la Vida. \\ Av. Gral. Rumiñahui s/n. Sangolquí, Ecuador
}

\begin{abstract}
Resumen
El propósito de esta investigación fue evaluar el efecto de asociar especies vegetales como cobertura viva en suelos cultivados con uvilla (Physalis peruviana L), para evitar la evaporación, controlar la erosión y mejorar el aporte nutricional al frutal; los tratamientos evaluados fueron: T1 alfalfa (Medicago sativa), T2 trébol blanco (Trifolium repens), T3 raigrás (Loliun perenne), T4 raigrás + trébol, T5 testigo (manejo convencional) distribuidos en un diseño de bloques completamente al azar (cuatro repeticiones). Las variables analizadas fueron: prendimiento de la uvilla, altura de planta, diámetro de tallo principal, área foliar, rendimiento, calidad del fruto, nivel de erosión y contenido de nitrógeno en el suelo. Los resultados indicaron que en el tratamiento T1 cuya cobertura vegetal fue la alfalfa, la uvilla alcanzó un rendimiento de 7,83 tn/ha, en tanto que en el T3 cuya cobertura vegetal fue el raigrás la uvilla alcanzó $0,52 \mathrm{tn} / \mathrm{ha}$. En el caso del nivel de erosión del suelo, los tratamientos más destacados fueron: T3 y T4, los cuales recibieron menos labores de escarda y tuvieron mayor capacidad de cubrimiento del suelo, aunque compitieron de manera agresiva por nutrientes (especialmente nitrógeno) y espacio con la uvilla, lo que afectó su rendimiento; de ahí que el T1 alfalfa, es el más recomendable ya que no presentó diferencias estadísticas en cuanto a la erosión, además aportó nitrógeno, a través del proceso de fijación biológica, lo que favorece el reciclaje de nutrientes en el sistema agrícola y genera un espacio adecuado para el desarrollo del frutal.
\end{abstract}

Palabras clave: uvilla, cobertura vegetal, alfalfa-uvilla, frutal andino.

\begin{abstract}
The purpose of the research was to evaluate the effect of plant species associate as cover soils cultivated with uvilla (Physalis peruviana L), to prevent evaporation, erosion and improve the nutritional contribution to fruit. The studied factor was the living mulch and soil treatments were evaluated: T1 alfalfa (Medicago sativa), T2 white clover (Trifolium repens), T3 ryegrass (Perennial loliun), ryegrass + clover T4, T5 control (conventional management) distributed in a design of a randomized complete block (four repetitions). The variables analyzed were: arrest of uvilla, height, main stem diameter, leaf area, yield, fruit quality, erosion levels and nitrogen content in the soil. The results indicated that treatment was T1 whose plant cover alfalfa, uvilla performance reached $7.83 \mathrm{t} /$ ha, while in Q3 which was the ryegrass plant cover the uvilla reached 0.52 tons / ha. In the case of soil erosion, the leading treatments were: T3 and T4, which received less hoeing and had greater ability to cover the ground, although they competed aggressively for nutrients (especially nitrogen) and space with
\end{abstract}


uvilla, which affected their performance; hence the T1 Alfalfa is the most recommended because it did not present statistical differences in erosion, and contributes nitrogen input, through the process of biological fixation, favoring nutrient cycling in the agricultural system and generates a suitable space for the development of fruit.

Keywords: Golden berry, vegetation cover, alfalfa-golden berry, andean fruit.

\section{Introducción}

La uvilla (Physalis peruviana L) es un frutal andino de características nutricionales especiales, posee altos contenidos de vitamina A (236 mg de B caroteno/100 g de fruto seco), vitamina C $(32,2 \mathrm{mg}$ de ácido ascórbico y $2.018 \mathrm{mg}$ de ácido cítrico/100 $\mathrm{g}$ de fruto seco), complejo $\mathrm{B}$, minerales como hierro $(\mathrm{Fe})$ y fósforo $(\mathrm{P})$, pero además se le reconocen propiedades medicinales (Mora et al., 2006). La uvilla es una fruta exótica de buena acogida en los mercados internacionales. La temperatura óptima requerida para el cultivo de uvilla oscila entre los 13 y $16^{\circ} \mathrm{C}$ y necesita 1.500 a 2.000 horas de luz solar directa, condiciones que se encuentran entre los 1800 y 2800 m s.n.m. (Angulo, 2011). La uvilla se ha cultivado bajo el sistema del monocultivo con alto consumo de insumos (Fischer et al., 2014), lo cual genera varios efectos negativos en los agroecosistemas (Altamirano, 2010).

El control mecánico de las malezas en la producción de uvilla es una práctica cultural que deja al suelo descubierto y sometido a procesos de erosión. Una alternativa a la remoción del suelo para control de malezas es la implementación de cultivos asociados, que actúen como cobertura viva del suelo y prevengan la erosión. Además, la utilización de cultivos asociados trae consigo otros beneficios como: aporte de nitrógeno $(\mathrm{N})$ e incorporación de materia orgánica $(\mathrm{MO})$ que mejoran las propiedades físico-químicas y biológicas del suelo, controlan la erosión, promueven el ciclo natural de nutrientes y el control natural de plagas. Es importante seleccionar de manera adecuada especies que se asocien como coberturas vegetales, así como conocer su manejo, pues las especies escogidas deben poseer espacio suficiente para su desarrollo (Altieri, 2009). La presente investigación tuvo como fin evaluar el uso de una asociación de coberturas vegetales vivas en el control de la erosión, en el mejoramiento del ciclo del $\mathrm{N}$ y en el incremento del rendimiento y productividad de los cultivos.

\section{Metodología}

\subsection{Ubicación política y geográfica de la investigación}

La presente investigación se la realizó en la hacienda "San Francisco", ubicada en la parroquia Huaca, cantón San Pedro de Huaca, provincia del Carchi. El predio está localizado a $00^{\circ} 38^{\prime} 29^{\prime \prime}$ latitud Norte y $77^{\circ} 43^{\prime} 35^{\prime}$ ' longitud Oeste; a una altitud de 2.830 m s.n.m., caracterizado por presentar temperaturas promedio anuales de $11{ }^{\circ} \mathrm{C}$ y $1.100 \mathrm{~mm}$ de precipitación anual (Mora Quilismal, 2013).

\subsection{Factores en estudio}

Los factores de estudio en la presente investigación fueron cinco diferentes coberturas vegetales vivas del suelo sobre las cuales se cultivó uvilla. En la Tabla 1 se presenta la descripción de los tratamientos.

Tabla 1. Tratamientos en estudio.

\begin{tabular}{ccc}
\hline Tratamientos & Tipo de cobertura & Alcance de la cobertura (\%) \\
\hline T1 & Alfalfa (Medicago sativa) & $100 \%$ \\
T2 & Trébol blanco (Trifolium repens) & $100 \%$ \\
T3 & Raigrás (Loliun perenne) & $100 \%$ \\
T4 & Raigrás (Loliun perenne) + trébol (Trifolium repens) & $75 \%+25 \%$ \\
T5 & Sin cobertura (manejo convencional o testigo) & \\
\hline
\end{tabular}




\subsection{Descripción del experimento}

Se utilizó un diseño experimental de bloques completamente al azar (DBCA) con 5 tratamientos, 4 repeticiones y 20 unidades experimentales. El área total del experimento fue de $2.912 \mathrm{~m}^{2}$; dividida en parcelas de $120 \mathrm{~m}^{2}$ y un área neta de evaluación de $80 \mathrm{~m}^{2}$. Las especies vegetales utilizadas para las coberturas se sembraron a una densidad de $2 \times 2 \mathrm{~m}$, para un total de 30 plantas por parcela total y 20 plantas por parcela neta. Las especies utilizadas fueron seleccionadas por su gran capacidad de adaptación a las condiciones edafo-climáticas de la zona donde está el sitio experimental y por su potencial de fijación de $\mathrm{N}$ atmosférico y gran desarrollo de biomasa

\subsection{Análisis estadístico}

Se realizó el análisis de varianza (ANOVA) y prueba de Tukey para comparación de medias, utilizando el software estadístico INFOSTAT, versión libre (Herrera, 1999).

\subsection{Variables medidas}

Luego de que se establecieron las coberturas en las diferentes parcelas experimentales, se realizó la siembra del cultivo de la uvilla. Se procedió luego a la evaluación del efecto de los tratamientos de cobertura en las siguientes variables de crecimiento y producción de la uvilla, así como el efecto de las coberturas en la reducción de la erosión. Prendimiento del cultivo de uvilla: se determinó el porcentaje de prendimiento mediante conteo de plantas de la parcela neta, a los 15 días después del trasplante (15 ddt).

- Diámetro de tallo principal en el cultivo de uvilla: se identificó el diámetro $(\mathrm{mm})$ del tallo principal a $5 \mathrm{~cm}$ de altura; desde la base de la planta.

- $\quad$ Altura de planta: se determinó la altura $(\mathrm{cm})$ de la planta desde la base al ápice a los 30, 120 y 240 ddt.

- Área foliar (por planta) en el cultivo de la uvilla: se estimó el área foliar contabilizando el número total de hojas de la planta y midiendo su largo $(\mathrm{cm})$ y mediante un modelo matemático, se estimó el área foliar total.

- Rendimiento de frutos de uvilla: se pesó la producción total de fruta en cada unidad experimental; y se transformó el peso a $(\mathrm{kg} /$ ha). Se evaluó el peso de la producción con cáliz y sin cáliz.

- Peso y forma del fruto: se determinó el peso del fruto y su diámetro ecuatorial y polar.

- Erosión del suelo: esta variable se determinó en cada unidad experimental mediante el método de varillas que consiste en medir el desplazamiento del suelo a lo largo de la columna (varilla) y luego se calculó la pérdida total de suelo en $\mathrm{T} / \mathrm{h}$.

- Contenido de $\mathrm{N}$ en el suelo: se determinó el contenido de $\mathrm{N}(\%)$ en el suelo de cada una de las parcelas experimentales mediante el método de Kjeldahl.

\section{Resultados y discusión}

\subsection{Prendimiento del cultivo de uvilla bajo el efec- to de la asociación de coberturas vegetales}

La evaluación del efecto de las diferentes coberturas en el prendimiento de las uvillas indicó que existieron diferencias estadísticas significativas entre tratamientos. El porcentaje de prendimiento más alto se registró en los tratamientos: T5 y $\mathrm{T} 1$ con $97,5 \mathrm{~s} \%$ de plantas prendidas (Tabla 2). En estos tratamientos la competencia por espacio fue menor que en el resto. Una desventaja de las coberturas vegetales puede ser la competencia agresiva por espacio y nutrientes, la misma que fue más acentuada en los tratamientos que tienen como base raigrás. Borges et al. (2015) encontraron que el adecuado manejo de las coberturas vegetales favorece el desarrollo radicular y prendimiento de plantas frutales, resultados que tienen similitud con el efecto que pudo observarse en la cobertura de alfalfa sobre el cultivo de uvilla. 
Tabla 2. Efecto de diferentes coberturas vegetales sobre el porcentaje de prendimiento de uvilla.

\begin{tabular}{ccc}
\hline Tratamientos & Descripción & $\begin{array}{c}\text { Medias de porcentaje } \\
\text { de prendimiento }\end{array}$ \\
\hline T1 & Alfalfa (Medicago sativa) & $\mathbf{9 7 , 5} \mathbf{a}^{*}$ \\
T2 & Trébol blanco (Trifolium repens) & $95,0 \mathrm{ab}$ \\
T3 & Raigrás (Loliun perenne) & $82,5 \mathrm{ab}$ \\
T4 & Raigrás (Loliun perenne) + trébol (Trifolium repens) & $77,5 \mathrm{~b}$ \\
T5 & Sin cobertura (manejo convencional o testigo) & $97,5 \mathrm{a}$ \\
* Valores con diferente letra difieren estadísticamente según la prueba de Tukey al 5 \%. & \\
\hline
\end{tabular}

3.2. Altura de planta en el cultivo de uvilla bajo el efecto de la asociación de coberturas vegetales

El análisis de varianza para altura de planta realizado a los 30 ddt no detectó diferencias estadísticas en el experimento, la media del experimento en esta etapa inicial fue de $11,26 \mathrm{~cm} /$ planta, sin embargo, las mediciones de la altura de la planta a los 120 y 240 ddt identificaron diferencias estadísticas entre tratamientos. La prueba de Tukey para altura de planta a los 240 ddt destacó el comportamiento de la altura de planta en T1 que logró un promedio de $115,2 \mathrm{~cm} /$ planta, diferenciándose del resto de tratamientos. Se nota que el aporte de $\mathrm{N}$ de la alfalfa mejora el desarrollo de la planta de uvilla, diferente de lo ocurrido con las coberturas vegetales con raigrás, donde se observó que el desarrollo de la planta fue menor, ya que las coberturas compitieron por nutrientes con las plantas de uvilla (Tabla 3). Los resultados encontrados en este ensayo coinciden con lo demostrado por Miller et al. (1996) quienes determinaron el efecto positivo de las leguminosas como coberturas de suelo en el desarrollo de las plantas frutales y los efectos negativos de las poáceas.

Tabla 3. Efecto de diferentes coberturas vegetales sobre el porcentaje de altura (cm) de la planta de uvilla.

\begin{tabular}{|c|c|c|c|c|}
\hline Tratamientos & Descripción & $30 \mathrm{ddt}$ & $120 \mathrm{ddt}$ & $240 \mathrm{ddt}$ \\
\hline T1 & Alfalfa (Medicago sativa) & 11,40 & $28,32 b^{*}$ & $115,20 \mathrm{a}$ \\
\hline $\mathbf{T 2}$ & $\begin{array}{l}\text { Trébol Blanco } \\
\text { (Trifolium repens) }\end{array}$ & 10,70 & $41,77 \mathrm{a}$ & $77,95 \mathrm{ab}$ \\
\hline T3 & Raigrás (Loliun perenne) & 12,40 & $25,42 \mathrm{~b}$ & $52,45 \mathrm{~b}$ \\
\hline T4 & $\begin{array}{c}\text { Raigrás (Loliun perenne) }+ \\
\text { trébol (Trifolium repens) }\end{array}$ & 11,70 & $23,50 \mathrm{~b}$ & $67,35 \mathrm{ab}$ \\
\hline T5 & $\begin{array}{l}\text { Sin cobertura (manejo } \\
\text { convencional o testigo) }\end{array}$ & 9,90 & $25,62 \mathrm{~b}$ & $78,60 \mathrm{ab}$ \\
\hline
\end{tabular}

\subsection{Diámetro de tallos principales en el cultivo} de uvilla bajo el efecto de la asociación de coberturas vegetales

Los resultados encontrados mediante un análisis de varianza relacionado con la variable diámetro de tallo principal, establecieron que en la etapa inicial (30 ddt) los tratamientos no se diferenciaron estadísticamente, esto se debió a que el cultivo se encontraba en etapa de establecimiento y aún no se evidenciaba el efecto de los tratamientos; a partir de los 120 ddt existió diferencias estadísticas marcadas en los tratamientos (1\%), que influyeron en el grosor del tallo, tendencia que se mantuvo hasta los 240 ddt, la media del experimento en esta etapa fue de $1.86 \mathrm{~cm} /$ tallo. 
En la Tabla 4 se observa que a los $120 \mathrm{ddt}$ se destacó el T1 con $0,86 \mathrm{~cm} /$ tallo principal, el cual se ubicó en el rango A según la prueba de Tukey, superando al resto de tratamientos. Esta tendencia se manifestó hasta el final del cultivo (240 ddt), donde el T1 alcanzó un diámetro final de $2,3 \mathrm{~cm} /$ tallo principal, teniendo a la alfalfa como la especie que presentó la mejor cobertura de suelo. Esta leguminosa mejoró el contenido de $\mathrm{N}$ en el suelo, optimizando la nutrición de los cultivos asociados y mejorando el desarrollo de los órganos de la planta (Campillo et al., 2003).

Borges et al. (2015) manifestaron que cuando existe un manejo adecuado de la asociación de coberturas vegetales se mejora las características físicas, químicas y microbiológicas del suelo, además que se evidencia mejor comportamiento en las características fenotípicas del cultivo, así lo comprobó trabajando con cítricos y papaya (Carica papaya), que al darles espacio adecuado, las plantas mejoraron su tamaño.

Tabla 4. Diámetro (cm) de tallos principales y prueba de Tukey en el cultivo de uvilla bajo el efecto de las coberturas vegetales.

\begin{tabular}{ccccccc}
\hline Tratamientos & $\mathbf{3 0} \mathbf{d d t}$ & $\begin{array}{c}\text { Error } \\
\text { Experimental }\end{array}$ & $\mathbf{1 2 0} \mathbf{d d t}$ & $\begin{array}{c}\text { Error } \\
\text { Experimental }\end{array}$ & $\mathbf{2 4 0} \mathbf{~ d d t}$ & Error Experimental \\
\hline $\mathrm{T} 1$ & 0,28 & $\pm 0,040$ & $0,86 \mathrm{a}$ & $\pm 0,047$ & $\mathbf{2 , 3 3} \mathbf{~ a b}$ & $\pm 0,23$ \\
$\mathrm{~T} 2$ & 0,27 & $\pm 0,015$ & $0,43 \mathrm{~b}$ & $\pm 0,033$ & $2,04 \mathrm{a}$ & $\pm 0,19$ \\
$\mathrm{~T} 3$ & 0,35 & $\pm 0,080$ & $0,46 \mathrm{~b}$ & $\pm 0,097$ & $1,54 \mathrm{ab}$ & $\pm 0,07$ \\
$\mathrm{~T} 4$ & 0,27 & $\pm 0,013$ & $0,34 \mathrm{~b}$ & $\pm 0,012$ & $1,32 \mathrm{~b}$ & $\pm 0,09$ \\
$\mathrm{~T} 5$ & 0,24 & $\pm 0,016$ & $0,53 \mathrm{~b}$ & $\pm 0,093$ & $2,07 \mathrm{ab}$ & $\pm 0,29$ \\
\hline
\end{tabular}

\section{4. Área foliar (por planta) en el cultivo de uvilla bajo el efecto de asociación de coberturas vegetales}

Los resultados encontrado mediante un análisis de varianza para la variable área foliar en el cultivo de uvilla, registraron diferencias estadísticas entre tratamientos a un nivel del $1 \%$ durante todo el experimento; a los 240 ddt el cultivo de uvilla registró un área promedio de $5.117,27 \mathrm{~cm}^{2} /$ planta.

La prueba de Tukey (Tabla 5), indicó que al inicio hubo diferencias mínimas entre los tratamientos, hasta los 30 ddt, después de lo cual se empezó a evidenciar diferencias marcadas, resaltando el $\mathrm{T} 1 \mathrm{como}$ el mejor para asociar el cultivo; en tanto que el T4 fue el de menor respuesta, lo que indicó que el raigráss + trébol es una mezcla competitiva con el cultivo de uvilla, pues no favoreció el desarrollo foliar y tuvo afectaciones negativas en el rendimiento; el tratamiento testigo con un manejo convencional también tuvo un buen desarrollo foliar, el cual consistió en eliminar la competencia que ejercen las plantas no deseadas al cultivo de interés, arvenses que fueron incorporadas al suelo y aportan materia orgánica al mismo (Fischer et al., 2005).

En esta misma tabla, se observa que al final el desarrollo foliar (área) de la planta de uvilla, presentó un efecto significativo ante la asociación de coberturas vegetales a partir de los 120 días de establecido, lo cual continuó hasta el final del experimento (240 ddt); donde la alfalfa se destacó por ejercer mayor influencia en el crecimiento de la variable estudiada, donde su desarrollo fue más acelerado frente a los otros tratamientos. Este efecto pudo deberse a que la alfalfa, al poseer mayor área radicular, es más amplia la exploración de las raíces en el suelo, además que contribuyó con un mayor aporte en la fijación de $\mathrm{N}$ y aeración al cultivo, lo cual favoreció al desarrollo foliar de la uvilla, esto no sucedió con el trébol, que a pesar de ser una leguminosa no se comportó con eficiencia en la fijación de $\mathrm{N}$, debido a que sus raíces poseen menor capacidad de exploración en el suelo. 
Tabla 5. Área foliar ( $\mathrm{cm}^{2} /$ planta) y prueba de Tukey para el cultivo de uvilla bajo efecto de la asociación de coberturas vegetales

\begin{tabular}{ccccccc}
\hline Tratamientos & $\mathbf{3 0} \mathbf{d d t}$ & $\begin{array}{c}\text { Error } \\
\text { Experimental }\end{array}$ & $\mathbf{1 2 0} \mathbf{d d t}$ & $\begin{array}{c}\text { Error } \\
\text { Experimental }\end{array}$ & $\mathbf{2 4 0 ~ d d t}$ & $\begin{array}{c}\text { Error } \\
\text { Experimental }\end{array}$ \\
\hline $\mathrm{T} 1$ & $454,56 \mathrm{a}$ & $\pm 45,82$ & $3.209,34 \mathrm{~b}$ & $\pm 64,09$ & $\mathbf{5 . 1 1 7 , 2 7} \mathbf{a}$ & $\pm 283,29$ \\
$\mathrm{~T} 2$ & $130,08 \mathrm{a}$ & $\pm 5,62$ & $1.311,94 \mathrm{a}$ & $\pm 180,21$ & $2.271,18 \mathrm{ab}$ & $\pm 474,88$ \\
$\mathrm{~T} 3$ & $110,75 \mathrm{~b}$ & $\pm 8,80$ & $359,61 \mathrm{c}$ & $\pm 48,94$ & $962,96 \mathrm{bc}$ & $\pm 174,82$ \\
$\mathrm{~T} 4$ & $95,81 \mathrm{~b}$ & $\pm 3,84$ & $350,37 \mathrm{c}$ & $\pm 78,41$ & $842,05 \mathrm{c}$ & $\pm 199,92$ \\
$\mathrm{~T} 5$ & $221,94 \mathrm{~b}$ & $\pm 80,55$ & $1.523,31 \mathrm{ab}$ & $\pm 460,67$ & $2.700,26 \mathrm{ab}$ & $\pm 1071,99$ \\
\hline
\end{tabular}

3.5. Peso del fruto en el cultivo de uvilla bajo el efecto de la asociación de coberturas vegetales

Los resultados encontrados mediante el análisis de varianza para el peso del fruto indicaron que existen diferencias estadísticas al $1 \%$ para tratamientos y del $5 \%$ entre repeticiones, el peso promedio de frutos fue de $6,67 \mathrm{~g} /$ fruto.

La prueba de Tukey al $5 \%$ para el peso del fruto (Tabla 6) evidenció al tratamiento T1 con el mayor peso del fruto de uvilla, con respecto al resto de tratamientos obteniéndose un peso final a los 240 ddt de $8,05 \mathrm{~g} /$ fruto; situación contraria al T4, donde se registró el peso más bajo de frutos (4,6 gr/fruto); es impor- tante destacar que la asociación de las coberturas vegetales como el raigrás compiten agresivamente con la planta de uvilla por espacio, nutrientes (en especial $\mathrm{N}$ ), agua y otros factores, lo cual afecta el desarrollo del fruto. El peso de frutos obtenido en este ensayo, es superior al registrado por Fischer et al. (2005), quienes obtuvieron un promedio de 5,7 g/fruto;

Sandoval (2005), obtuvo resultados similares de Fisher et al. (20015) en cuanto a la variable peso de fruto, al trabajar con asociaciones de coberturas vegetales en cítricos. Este autor recomendó controlar la competencia del espacio y nutrientes que se genera entre las coberturas vegetales asociados a cultivos para no afectar su desarrollo.

Tabla 6. Prueba de Tukey al 5\% para el peso del fruto en el cultivo de uvilla

\begin{tabular}{ccc}
\hline Tratamientos & g/fruto & Error Experimental \\
\hline T1 & $\mathbf{8 , 0 5} \mathbf{a}$ & $\pm 0,26$ \\
T2 & $7,84 \mathrm{ab}$ & $\pm 1,39$ \\
$\mathrm{~T} 3$ & $5,05 \mathrm{ab}$ & $\pm 0,72$ \\
$\mathrm{~T} 4$ & $4,60 \mathrm{~b}$ & $\pm 0,82$ \\
$\mathrm{~T} 5$ & $7,81 \mathrm{ab}$ & $\pm 1,40$ \\
\hline
\end{tabular}

3.6. Diámetro ecuatorial y polar del fruto de uvilla bajo el efecto de la asociación de coberturas vegetales

Mediante los resultados encontrados al realizar un análisis de varianza para la variable diámetro ecua- torial y polar del fruto de la uvilla generaron diferencias estadísticas entre tratamientos y entre repeticiones. El diámetro ecuatorial promedio del fruto en el experimento fue de $21,01 \mathrm{~mm} /$ fruto y el diámetro polar promedio de fruto en el experimento fue de 21,71 $\mathrm{mm} /$ fruto a los $240 \mathrm{ddt}$. 
La prueba de Tukey al 5\% para el diámetro ecuatorial final (240 ddt) del fruto de uvilla, destacó al T1 con un diámetro de $26 \mathrm{~mm} /$ fruto, seguido del T5 con 23,50 mm / fruto; se debe tomar en cuenta que este último también se destacó en cuanto a variables como altura de planta y área foliar. Las labores culturales realizadas en este tratamiento, permitieron airear el suelo y favorecer el crecimiento del frutal y obtención de un fruto con buenas características de calidad (Tabla 7).

Tabla 7. Prueba de Tukey al 5\% para el diámetro ecuatorial y diámetro polar del fruto ( $\mathrm{mm} /$ fruto) en el cultivo de uvilla a $\operatorname{los} 240 \mathrm{ddt}$.

\begin{tabular}{ccccc}
\hline Tratamientos & Diámetro Ecuatorial & Error Experimental & Diámetro Polar & Error Experimental \\
\hline $\mathrm{T} 1$ & $\mathbf{2 6 , 0 0} \mathbf{a}$ & $\pm 1,35$ & $\mathbf{2 6 , 6 5} \mathbf{~ a}$ & $\pm 2,04$ \\
$\mathrm{~T} 2$ & $18,60 \mathrm{ab}$ & $\pm 2,56$ & $19,65 \mathrm{ab}$ & $\pm 2,33$ \\
$\mathrm{~T} 3$ & $20,50 \mathrm{ab}$ & $\pm 2,07$ & $20,65 \mathrm{ab}$ & $\pm 2,49$ \\
$\mathrm{~T} 4$ & $16,40 \mathrm{~b}$ & $16,33 \mathrm{~b}$ & $\pm 3,01$ \\
$\mathrm{~T} 5$ & $23,50 \mathrm{ab}$ & $\pm 3,97$ & $25,25 \mathrm{a}$ & $\pm 2,17$ \\
\hline
\end{tabular}

La prueba de Tukey al $5 \%$ para el diámetro polar del fruto en el cultivo de uvilla, destacó al T1 (26,65 mm/fruto) y lo ubicó en el rango A junto con el T5 (25,25 mm/fruto); en el último rango (B) se encontró el T4, el mismo que también en las variables altura y área foliar, ocupa rangos inferiores.

Fischer et al. (2005), obtuvieron un promedio de $21,71 \mathrm{~mm} /$ fruto, para el diámetro ecuatorial y $21,01 \mathrm{~mm} /$ fruto para el diámetro polar, resultados que se encuentran en el rango de esta variable y que fueron obtenidos en este ensayo.

\subsection{Rendimiento en el cultivo de uvilla bajo el efecto de coberturas vegetales}

Los resultados obtenidos mediante un análisis de varianza para el rendimiento en el cultivo de uvilla con cáliz y sin cáliz, presentaron diferencias estadísticas al $1 \%$ entre tratamientos. El promedio de rendimiento del experimento fue de
2,81 $\mathrm{T} / \mathrm{ha}$ (frutos con cáliz) y $2,53 \mathrm{~T} / \mathrm{ha}$ (frutos sin cáliz).

La prueba de Tukey al $5 \%$ para el rendimiento, tanto con cáliz como sin cáliz en el cultivo de uvilla (Tabla 8) generó dos rangos: el A, donde se ubicó el $\mathrm{T} 1$ con $7,83 \mathrm{~T} / \mathrm{ha}$ para el rendimiento con cáliz y 6,91 T/ha para el rendimiento sin cáliz y en el Grupo $\mathrm{B}$, donde se ubicaron el resto de tratamientos con rendimientos inferiores al anterior. Los tratamientos que poseen una asociación de coberturas vegetales con raigrás obtuvieron los rendimientos más bajos del experimento, pues la competencia por nutrientes entre la uvilla y la cobertura vegetal resultó ser muy agresiva, en este caso, los dos cultivos asociados a manera de cobertura vegetal, extraen $\mathrm{N}$ del suelo para su nutrición. Mientras el raigrás tenga disponibilidad de $\mathrm{N}$ (requerimiento $40 \mathrm{~kg} / \mathrm{N} /$ ), mejorará su desarrollo (Bailleres et al., 2010). El tratamiento a base de alfalfa desarrolló más follaje que el resto de tratamientos mejorando su capacidad fotosintética, que se evidenció en mayor producción de biomasa.

Tabla 8. Prueba de Tukey al 5\% para el rendimiento (T/ha) en el cultivo de uvilla.

\begin{tabular}{ccccc}
\hline Tratamientos & Rendimiento con cáliz & Error Experimental & Rendimiento sin cáliz & Error Experimental \\
\hline T1 & $\mathbf{7 , 8 3}$ a & $\pm \mathbf{0 , 1 7}$ & $\mathbf{6 , 9 1} \mathbf{a}$ & $\pm \mathbf{0 , 1 4}$ \\
$\mathrm{T} 2$ & $1,94 \mathrm{~b}$ & $\pm \mathbf{0 , 6 1}$ & $1,88 \mathrm{~b}$ & $\pm \mathbf{0 , 5 3}$ \\
$\mathrm{T} 3$ & $0,52 \mathrm{~b}$ & $\pm \mathbf{1 , 1 0}$ & $0,43 \mathrm{~b}$ & $\pm \mathbf{1 , 0 6}$ \\
$\mathrm{T} 4$ & $1,07 \mathrm{~b}$ & $\pm \mathbf{1 , 0 9}$ & $2,96 \mathrm{~b}$ & $\pm \mathbf{0 , 9 4}$ \\
$\mathrm{T} 5$ & $2,71 \mathrm{~b}$ & $\pm \mathbf{0 , 4 6}$ & $\pm \mathbf{0 , 4 2}$ \\
\hline
\end{tabular}


La cobertura vegetal a base de la asociación de alfalfa con uvilla, influyó positivamente en el desarrollo del frutal y en su rendimiento, pues el aporte de $\mathrm{N}$ de esta leguminosa a la asociación con el cultivo, evitó las deficiencias de este nutriente determinante para las plantas. Es importante destacar lo que demostró Borges et al. (2007), en sus experiencias con leguminosas, donde sus raíces profundas promueven el reciclaje de nutrientes y evitan su lixiviación, manteniéndoles así, disponibles para los cultivos.

\subsection{Contenido de nitrógeno $(N)$ en el suelo}

Los resultados obtenidos mediante un análisis de varianza para conocer el contenido de $\mathrm{N}$ total del suelo muestran diferencias estadísticas para repeticiones a un nivel del $5 \%$, mas no para tratamientos, esto indica que fue necesario su bloqueo en el experimento con el fin de disminuir el error experimental. El contenido promedio de $\mathrm{N}$ inicial (antes de instalar el experimento) en el suelo fue de $0,658 \%$ y al final del experimento fue de $0,526 \%$; al comparar el contenido de $\mathrm{N}$ del suelo de la evaluación inicial con la final se observa que el contenido disminuyó en el trascurso del experimento, dependiendo de cada tratamiento.

Comparando el contenido inicial de $\mathrm{N}$ del suelo con el del final de la investigación (240 ddt) se determinó una menor pérdida de este elemento en los tratamientos siguientes: T1 con una pérdida de $0,07 \%$ y el $\mathrm{T} 2$ con $0,082 \%$ de N perdido; al contrario de aquellos tratamientos que evidenciaron una mayor pérdida de $\mathrm{N}$ : T5 con $0,124 \%$ de N, T3 con 0,182 de $\mathrm{N}$ y el T4 con $0,230 \%$ de $\mathrm{N}$; hay que tomar en cuenta que la asociación de las coberturas vegetales a base de alfalfa (T1), y trébol (T2) aportaron $\mathrm{N}$ al agroecosistema.

Esto determinó que la pérdida o consumo de $\mathrm{N}$ por el cultivo de uvilla en el T1 y T2 no sufrió considerables disminuciones; contrario a los tratamientos que en su cobertura vegetal no poseen o es mínima la presencia de leguminosas (T4) la pérdida de $\mathrm{N}$ del suelo es alta y afecta negativamente el crecimiento y rendimiento de este fruto andino que también consume este elemento del suelo (Tabla 9). Resultados similares fueron descritos por Aruani et al. (2006) y Campillo et al. (2003).

Tabla 9. Contenido de $\mathrm{N}$ total del suelo (\%) en el cultivo de uvilla a los $240 \mathrm{ddt}$.

\begin{tabular}{cccccc}
\hline Tratamientos & $\begin{array}{c}\text { Contenido } \\
\text { Inicial N }\end{array}$ & $\begin{array}{c}\text { Error } \\
\text { Experimental }\end{array}$ & $\begin{array}{c}\text { Contenido } \\
\text { Final de N }\end{array}$ & $\begin{array}{c}\text { Error } \\
\text { Experimental }\end{array}$ & $\begin{array}{c}\text { Diferencia del } \\
\text { contenido de N }\end{array}$ \\
\hline T1 & 0,622 & $\pm 0,058$ & 0,615 & $\pm 0,015$ & $\mathbf{- 0 , 0 7 0}$ \\
T2 & 0,640 & $\pm 0,067$ & 0,558 & $\pm 0,023$ & $-0,082$ \\
T3 & 0,685 & $\pm 0,026$ & 0,503 & $\pm 0,053$ & $-0,124$ \\
T4 & 0,685 & $\pm 0,029$ & 0,455 & $\pm 0,069$ & $-0,230$ \\
T5 & 0,647 & $\pm 0,094$ & 0,523 & $\pm 0,044$ & $-0,137$ \\
\hline
\end{tabular}

\subsection{Erosión de suelo en el cultivo de uvilla bajo el efecto de la asociación de coberturas vegetales}

Los resultados encontrados mediante un análisis de varianza para la erosión del suelo (240 ddt) mostró diferencias estadísticas entre tratamientos a un nivel del $1 \%$, la erosión promedio del experimento fue de 7,6 T/ha, considerándose una erosión moderada de grado 3 según la escala generada por la FAO.

En la Tabla 10, se observan los resultados obtenidos para la variable erosión del suelo, mediante la prueba de Tukey al 5\%, donde el T5 registró una erosión de 14,57 T/ha y el T2 12,13 T/ha; en estos dos tratamientos se realizó una mayor actividad con labores de escarda en el cultivo. Al res- pecto Huerta (2007) manifiestó que a medida que se aumenta las actividades de escarda disminuye la eficiencia en el control de la erosión del suelo. En cambio, el tratamiento 1 (con alfalfa) tuvo una pérdida de suelo por erosión de $6,77 \mathrm{~T} / \mathrm{ha}$, a pesar de las labores culturales a las que estuvo expuesta (escarda); en los tratamientos, T3 y T4 fue donde se generó menor erosión de suelo, debido a que fueron intervenidos mínimamente con labores de escarda; además, es importante resaltar que la asociación de coberturas vegetales con alfalfa y raigrás en esta variable de erosión del suelo, son menos proclives a la pérdida de suelo por poseer un buen cubrimiento del suelo y buena masa radicular, así lo ratificaron Cerda et al. (2007) y Verde (2013) en sus investigaciones. 
Tabla 10. Prueba de Tukey al $5 \%$ para la erosión del suelo en el cultivo de uvilla a los $240 \mathrm{ddt}$.

\begin{tabular}{ccc}
\hline Tratamientos & Medias (T/ha) & Error Experimental \\
\hline T5 & $\mathbf{1 4 , 5 7 ~ a ~}$ & $\pm 4,4$ \\
T2 & $12,13 \mathrm{ab}$ & $\pm 1,84$ \\
T1 & $6,77 \mathrm{ab}$ & $\pm 3,04$ \\
T3 & $2,58 \mathrm{~b}$ & $\pm 1,49$ \\
T4 & $1,97 \mathrm{~b}$ & $\pm 0,68$ \\
\hline
\end{tabular}

Es necesario enfatizar que en los tratamientos: T1, T2, T3 y T4, no se evidenciaron grandes diferencias estadísticas en la variable erosión de suelo. Así mismo se pudo evidenciar que los tratamientos a base de raigrás poseen un buen control para la erosión del suelo (Figura 1), aunque no favorecen el desarrollo óptimo del cultivo, ya que en estas asociaciones de las coberturas, de cierta manera se dificulta la aireación, el drenaje, la disponibilidad de nutrientes, condiciones necesarias para el desarrollo adecuado del cultivo. Estos resultados confirman lo establecido por Flores-López et al. (2012), quienes trabajaron sobre el control de la erosión mediante coberturas vegetales, utilizando diferentes especies de raigrás.

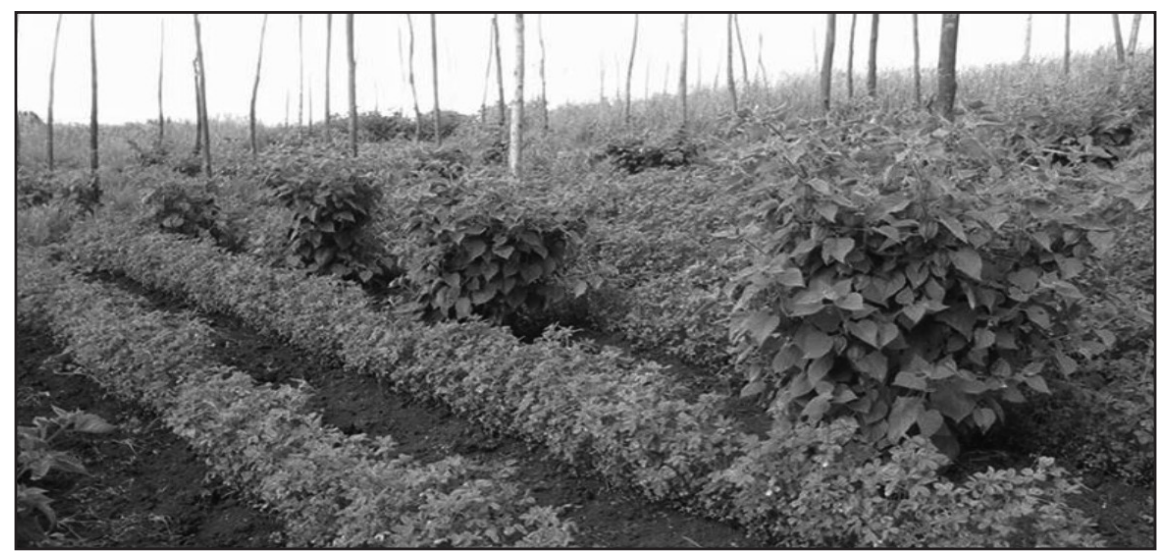

Figura 1. Uvilla en asociación con alfalfa.

\section{Conclusiones y recomendaciones}

- La asociación de coberturas vegetales en base a alfalfa, generó el mejor efecto en el desarrollo del cultivo, con un rendimiento de 7,86 T/ ha (con cáliz) y 6,91 T/ha (sin cáliz), desde los primeros cuatro meses y hasta el final del cultivo (240 ddt), superando al resto de tratamientos analizados.

- Mediante la utilización de coberturas vegetales en base a alfalfa (con escarda) y raigrás (sin escarda) se logró prevenir y controlar la erosión del suelo, comparado con el testigo absoluto (manejo convencional), donde se generó la más alta erosión del suelo, con 15,57 T/ha/año.
- La asociación de la uvilla con la alfalfa, como cobertura vegetal, mantuvo hasta un $90 \%$ del $\mathrm{N}$ total en el suelo, entre el inicio y el final del experimento.

- Se recomienda usar la asociación de cobertura vegetal a base de alfalfa con la uvilla, ya que esta leguminosa estimula su desarrollo y mejora sus rendimientos y calidad del fruto, gracias al aporte de $\mathrm{N}$ y a la promoción del reciclaje de nutrientes en el sistema agrícola.

- Se recomienda validar la cobertura vegetal a base de alfalfa en el cultivo de uvilla en una superficie más extensiva; así como también probarla en otras zonas geográficas y en diferentes tipos de suelo. 


\section{Referencias}

Altamirano, M. (2010). Estudio de la cadena pruductiva de la uvilla Physalis peruviana en la Sierra Norte del Ecuador. Quito: Universidad San Francisco de Quito.

Altieri, M. (2009). Desiertos verdes: monocultivos y sus impactos sobre la biodiversidad. México D.F: Gloobalhoy.

Angulo, R. (2011). Uchuva Physalis peruviana. Bogotá: Bayer CropScience.

Aruani, C., Sánchez, E., \& Reeb, P. (2006). Cambios en las propiedades de un suelo franco bajo producción orgánica de manzano utilizando coberturas vegetales. Cinecia del Suelo, vol. 24, n. 2, 131-137.

Bailleres, M., \& Pirodi, F. (2010). Sitio Argentino de Producción Animal. Obtenido de Fertilización en raigrás y avena: http://www.produccionanimal.com.ar/produccion_y_manejo_pasturas/ pasturas_fertilizacion/43-raigras_44.pdf

Borges, J., Leone, C., \& Oliveira , J. (2007). Manejo del suelo y coberturas vegetales en frutales experiencia en citricos y papaya en Brasil. Brazil: Embrapa y Fruticultura Tropical .

Borges, J., Leone, L., \& De Oliveiro, J. (20 de mayo de 2015). Manejo del suelo y coberturas vegetales en frutales - experienecia en citricos $y$ papaya en brasil. Obtenido de Biblioteca virtual de las ciencias en Cuba: http://www. bibliociencias.cu/gsdl/collect/revistas/index/ assoc/HASH0101/9e82e65d.dir/doc.pdf

Campillo , R., Urquiaga , S., Pino, I., \& Montenegro, A. (2003). Estimación de la fijación biológica de nitrógeno en leguminosas forrajeras mediante la metodología del 15n. Agricultura Técnica, vol. $63, n 2,169-179$.

Campillo, R., Urquiaga , S., Pino, I., \& Montenegro, A. (2003). Estimation of biological nitrogen fixation in forage legumes using a $15 \mathrm{~N}$ labeling methodology1. Agricultura Técnica, vol. $63 n$ 2, 169-179.

Cerdà, A., Bodí, M., \& Hevilla, E. (2007). Erosión del suelo en plantaciones de cítricos en laderas. Valle del río canyoles, Valencia. Agroecología 2, 85-91.

Fischer, G., Piedrahita, W., Miranda, D., \& Romero, J. (2005). Avances en cultivo, poscosecha y exportación de la uchuva
(Physalis peruviana L.) en Colombia. Bogotá: Universidad Nacional de Colombia.

Fischer, G., Almanza, P., \& Miranda, D. (2014). Importancia y cultivo de la uchuva (Physalis peruviana L.). Revista Brasileña de Fruticultura Vol.1(4):37.

Flores López, H., De la Mora , C., Ruiz Corral, J., \& Chávez Durán, Á. (2012). Efecto de la cobertura de suelo de tres cultivos sobre la erosión hídrica. Jalisco: Chapingo serie zonas áridas.

Herrera, J. (1999). Guía de aplicacion de pruebas estadisticas en el programa systat 7.0 para ciencias biologicas y forestales. Santa Cruz; Bolivia: BOLFOR.

Huerta, J. (2007). Efecto de la cobertura vegetal y raíces en la erosión del suelo. Texcoco: Colegio de Postgraduados.

Miller, P., Graves, W., Williams, W., \& Madson, B. (1996). Cultivos de cobertura para la agricultura de California. . California: Universidad de California, División de Agricultura.

Mora Quilismal, S. (2013). Planificación estratégica en la producción agropecuaria de la hacienda San Francisco de la Universidad Politécnica Estatal del Carchi. Ambato: UTA. 13 pgs.

Mora, R., Peña, A., López, E., Ayala, J., \& Ponce, D. (2006). Agrofenología de Physalis peruviana L. en invernadero y fertirriego. Revista Chapingo Serie Horticultura, 57-63.

Sandoval Rincón, J. (2005). Leguminosas como cobertura vegetal en el cultivo de los citricos. México: centro de desarrollo tecnológico y empresarial para frutales del trópico.

Verde, S. (2013). Inclusión de cultivos de cobertura en la rotación: experiencia de un productor. En C. Álvarez, A. Quiroga, D. Santos, \& M. Bodrero, Contribuciones de los cultivos de cobertura a la sostenibilidad de los sistemas de producción (pp. 165-169). La Pampa: Instituto Nacional de Tecnología Agropecuaria. 